\title{
Pregnancy Desire, Partner Serodiscordance, and Partner HIV Disclosure among Reproductive Age HIV-Infected Women in an Urban Clinic
}

\author{
Corinne M. Rhodes, ${ }^{1,2}$ Susan Cu-Uvin, ${ }^{3}$ and Aadia I. Rana $^{3}$ \\ ${ }^{1}$ General Internal Medicine, Massachusetts General Hospital, Harvard Medical School, Boston, MA 02114, USA \\ ${ }^{2}$ General Internal Medicine, Alpert Medical School of Brown University, Rhode Island Hospital, Providence, RI 02903, USA \\ ${ }^{3}$ Department of Medicine, Alpert Medical School of Brown University, The Miriam Hospital, Providence, RI 02906, USA
}

Correspondence should be addressed to Corinne M. Rhodes; crhodes@mgh.harvard.edu

Received 12 February 2016; Accepted 4 May 2016

Academic Editor: Charlene Dezzutti

Copyright ( 2016 Corinne M. Rhodes et al. This is an open access article distributed under the Creative Commons Attribution License, which permits unrestricted use, distribution, and reproduction in any medium, provided the original work is properly cited.

\begin{abstract}
Women comprise $25 \%$ of the US HIV epidemic, with many women of reproductive age. There is a need for providers to address the reproductive needs and desires of women with HIV given that effective antiretroviral therapy has transformed HIV into a chronic disease. This cross-sectional study shows high rates of partner serodiscordance (61\%) and moderate HIV disclosure to partners (61\%). Patients surveyed reported practitioners discuss condoms (94\%) and contraception (71\%) more often than pregnancy desire (38\%). In our sample, $44 \%$ of the surveyed women intended future pregnancy, whereas women who did not intend future pregnancy cited HIV/health and serodiscordance as the most common reasons (56\% and 35\%, resp.). There was no difference in the knowledge of mother-to-child transmission risk between women who intended or did not intend future pregnancy $(p=0.71)$. These results underline the need for provider training in reproductive counseling to promote risk reduction and education.
\end{abstract}

\section{Introduction}

There are 280,000 HIV-infected women and 200,000 heterosexual serodiscordant couples living in the United States, many of whom need reproductive health services $[1,2]$. Cross-sectional US studies report varied rates of pregnancy desire by women across time: from $29 \%$ in a nationally representative survey in 1998 [3] to $59 \%$ in an urban sample in 2010 [4]. A national sample of HIV-infected women from 2007 to 2009 [5] showed high rates (86.4\%) of unintentional pregnancy, suggesting that pregnancy counseling should extend to all HIV-infected women regardless of stated desire of future pregnancies.

According to recent US data, over half of the HIVinfected women are in serodiscordant relationships with similar reproductive desires to HIV-negative women [5-7]. One-third of women deny any discussion about reproductive health with their HIV provider $[5,7,8]$. This highlights the need for HIV providers to discuss strategies to reduce
HIV transmission including ensuring HIV suppression with antiretroviral therapy (ART), timed conception, and preexposure prophylaxis (PrEP) [4].

We seek to examine rates and impact of partner serodiscordance, partner HIV disclosure, practitioners' reproductive counseling, and mother-to-child transmission (MTCT) knowledge on HIV-infected women's reproductive desires at a large urban HIV clinic in New England.

\section{Methods}

A convenience sample of 100 English speaking adult HIVinfected women of reproductive age were enrolled from March 2012 to March 2014 at the Miriam Hospital Immunology Center, a large urban academic clinic serving 1600 HIVinfected patients in Rhode Island. Women were recruited consecutively by study personnel at clinic visits to complete an administered survey and were provided with a $\$ 10$ retail 
TABLE 1: Demographics and clinical information for women with HIV of reproductive age.

\begin{tabular}{|c|c|c|c|c|}
\hline & & $\begin{array}{c}\text { No reproductive } \\
\text { desire, } n=56\end{array}$ & $\begin{array}{l}\text { Desire for future } \\
\text { children, } n=44\end{array}$ & $p$ value \\
\hline Age: mean (SD) & Age at enrollment & $39.9(5.2)$ & $32.6(7.1)$ & $<0.0001$ \\
\hline \multirow{4}{*}{ Self-reported race } & White & $21(38 \%)$ & $11(25 \%)$ & \multirow{4}{*}{0.35} \\
\hline & Black & $12(21 \%)$ & $15(34 \%)$ & \\
\hline & Hispanic & $11(20 \%)$ & $11(25 \%)$ & \\
\hline & Other or multiple races & $12(21 \%)$ & $7(16 \%)$ & \\
\hline CD4: mean (SD) & Most recent & $588(311)$ & $586(289)$ & 0.97 \\
\hline AIDS & Self-reported AIDS & $11(20 \%)$ & $6(14 \%)$ & 0.44 \\
\hline \multirow{3}{*}{ ART status } & Yes & $52(93 \%)$ & $34(81 \%)$ & \multirow{3}{*}{0.13} \\
\hline & No, not indicated ${ }^{*}$ & $3(5 \%)$ & $3(7 \%)$ & \\
\hline & No, but indicated & $1(2 \%)$ & $5(12 \%)$ & \\
\hline \multirow{4}{*}{$\begin{array}{l}\text { Self-reported } \\
\text { HIV symptoms }\end{array}$} & None & $35(65 \%)$ & $31(70 \%)$ & \multirow{4}{*}{0.17} \\
\hline & Mild & $12(22 \%)$ & $4(9 \%)$ & \\
\hline & Moderate & $7(13 \%)$ & $9(20 \%)$ & \\
\hline & Severe & 0 & 0 & \\
\hline Parity: binary & Currently having child & $51(91 \%)$ & $32(73 \%)$ & 0.03 \\
\hline \multirow{3}{*}{ Parity: mean (SD) } & Pregnancies & $4.0(2.6)$ & $2.9(2.2)$ & 0.02 \\
\hline & Deliveries & $2.8(2.3)$ & $1.9(1.4)$ & 0.01 \\
\hline & Abortions & $20(36 \%)$ & $16(36 \%)$ & 1 \\
\hline
\end{tabular}

*ART indications at the time of data collection: CD4 count < 500, AIDS defining illness, pregnancy, acute opportunistic infections, Mycobacterium tuberculosis, HIV-associated nephropathy, and Hepatitis B coinfection when treatment is indicated [9].

gift card as an incentive. The survey is available as a supplemental file (Supplementary Material is available online at http://dx.doi.org/10.1155/2016/8048457). Exclusion criteria included current pregnancy, menopause (absent menses $>1$ year), or hysterectomy. The Miriam Hospital Institutional Review Board approved all study procedures.

Variables surveyed included self-reported demographics and clinical characteristics including ART use, sexual history (including condom and contraceptive use), sexual partners and HIV disclosure, self-defined HIV symptoms as either none, mild, moderate, or severe, and desire for future pregnancy. Women reported reasons for presence or absence of future reproductive desire as well as the effect of HIV, CD4 cell count/viral load (VL), and partner serodiscordance on their desire for future pregnancy. Participants reported previous discussions with providers about condom use, contraception, and desire for future pregnancy. Providers at the clinic include either internal medicine or obstetrics/ gynecology doctors. Participants were also asked a multiplechoice knowledge-based question about MTCT. CD4 count was collected by chart abstraction.

Results are reported using proportions, means, and standard deviations. Answer to "Do you desire to have more children in the future?" determined future reproductive desire. Continuous data were analyzed using two-tailed Student's $t$ tests and categorical data with Fisher's exact/chi squared tests with significance set at $0.05 \%$. Multivariable logistic regression was conducted using purposeful selection [6] with desire for future pregnancy as dependent variable by including candidates with $p$ values $<0.25$ (identified by univariate screen), sequentially removing nonconfounding variables with $p>0.10$, and testing each noncandidate for confounding. Results are reported in adjusted odds ratios (AOR) with 95\% confidence intervals (CI). We used SAS version 9.4 (SAS Institute Inc., Cary, NC) for statistical analyses.

\section{Results}

Of the 102 women who met inclusion criteria, 2 declined participation, citing time constraints. The mean age of this cohort was 36.7 , and self-reported race was $32 \%$ white, $27 \%$ black, $22 \%$ Hispanic, and 19\% multiple race, Asians, American Indians, or others. Table 1 compares demographic and clinical information in groups with and without desire for future pregnancy. Women desiring pregnancy were younger (32.6 versus 39.9, $p<0.0001)$ and reported less prior pregnancies (2.9 versus $4.0, p=0.02)$ and deliveries (1.9 versus 2.8 , $p=0.01)$. Other clinical markers including CD4 cell count, self-reported HIV symptoms, and AIDS diagnosis were not statistically different.

Table 2 reports sexual history, fertility desire, and provider communication. Most women in the study were sexually active (desire $64 \%$ versus no desire $74 \%, p=0.38$ ). There were high but similar rates of serodiscordant partnerships among sexually active women (desire $69 \%$ versus no desire $71 \%, p=0.77$ ) with both groups reporting that concern of infecting partner with HIV affected pregnancy plans (desire $51 \%$ versus no desire $33 \%, p=0.12$ ). Despite these concerns, only $20 \%$ of women with reproductive intent were 
TABLE 2: Sexual history, fertility desires, and provider communication for women with HIV of reproductive age.

\begin{tabular}{|c|c|c|c|c|}
\hline & & $\begin{array}{c}\text { No } \\
\text { reproductive } \\
\text { desire, } n=56\end{array}$ & $\begin{array}{l}\text { Desire for } \\
\text { future } \\
\text { children, } \\
n=44\end{array}$ & $p$ value \\
\hline \multirow{2}{*}{${ }^{*}$ Sexual activity } & Sexually active & $36(64 \%)$ & $32(74 \%)$ & \multirow{2}{*}{0.38} \\
\hline & Not sexually active & $20(36 \%)$ & $11(26 \%)$ & \\
\hline \multirow{3}{*}{$\begin{array}{l}{ }^{* *} \text { Partner's HIV } \\
\text { status }\end{array}$} & Known: HIV negative & $34(71 \%)$ & $27(69 \%)$ & \multirow{3}{*}{0.77} \\
\hline & Known: HIV positive & $10(21 \%)$ & $10(26 \%)$ & \\
\hline & Unknown & $4(8 \%)$ & $2(5 \%)$ & \\
\hline \multirow{3}{*}{$\begin{array}{l}{ }^{*} \text { Shared HIV status } \\
\text { with partner in the } \\
\text { last year }\end{array}$} & All & $28(57 \%)$ & $26(67 \%)$ & \multirow{3}{*}{0.53} \\
\hline & Some & $7(14 \%)$ & $6(15 \%)$ & \\
\hline & None & $14(29 \%)$ & $7(18 \%)$ & \\
\hline \multirow{5}{*}{${ }^{*}$ Condom use } & $100 \%$ & $33(59 \%)$ & $20(48 \%)$ & \multirow{5}{*}{0.11} \\
\hline & $75-99 \%$ & $6(11 \%)$ & $5(12 \%)$ & \\
\hline & $50-74 \%$ & $3(9 \%)$ & 0 & \\
\hline & $25-49 \%$ & $2(4 \%)$ & $3(7 \%)$ & \\
\hline & $<25 \%$ & $10(18 \%)$ & $14(33 \%)$ & \\
\hline \multirow{4}{*}{$\begin{array}{l}\text { Birth control } \\
\operatorname{method}(s)\end{array}$} & Barrier & $32(57 \%)$ & $20(45 \%)$ & 0.31 \\
\hline & Permanent & $15(27 \%)$ & $6(14 \%)$ & 0.14 \\
\hline & None/abstinence/natural & $18(32 \%)$ & $21(48 \%)$ & 0.15 \\
\hline & Hormonal contraceptive/long acting reversible contraception & $10(18 \%)$ & $10(23 \%)$ & 0.62 \\
\hline \multirow{9}{*}{ Fertility desire } & Do you desire to have children in the future? & 0 & $44(100 \%)$ & $<0.0001$ \\
\hline & Do you think you will have children in the future? & $2(4 \%)$ & $37(84 \%)$ & $<0.0001$ \\
\hline & Does your partner desire to have more children? & $6(13 \%)$ & $29(83 \%)$ & $<0.001$ \\
\hline & Are you currently having unprotected sex with the goal of becoming pregnant? & 0 & $10(23 \%)$ & $<0.0001$ \\
\hline & Has your HIV diagnosis impacted your plans regarding pursuing pregnancy? & $25(45 \%)$ & $20(45 \%)$ & 1 \\
\hline & $\begin{array}{c}\text { Have improvements in treatment and intervention made you desire more } \\
\text { children? }\end{array}$ & $6(11 \%)$ & $21(48 \%)$ & $<0.0001$ \\
\hline & Does your viral load or CD4 count affect your plans to pursue pregnancy? & $7(13 \%)$ & $8(18 \%)$ & 0.39 \\
\hline & $\begin{array}{c}{ }^{* * *} \text { Does your concern of infecting your (HIV negative) partner with HIV } \\
\text { affect your plans to pursue pregnancy? }\end{array}$ & $16(33 \%)$ & $19(51 \%)$ & 0.12 \\
\hline & $\begin{array}{l}\text { Have you considered or used alternative options (not unprotected sex) to } \\
\text { pursue pregnancy? }\end{array}$ & $4(7 \%)$ & $8(20 \%)$ & 0.11 \\
\hline \multirow{4}{*}{${ }^{*}$ MTCT rate } & $<1$ & $30(55 \%)$ & $29(66 \%)$ & \multirow{4}{*}{0.71} \\
\hline & $5-10 \%$ & $11(20 \%)$ & $7(16 \%)$ & \\
\hline & $10-20 \%$ & $3(5 \%)$ & $1(2 \%)$ & \\
\hline & $>25 \%$ & $11(20 \%)$ & $7(16 \%)$ & \\
\hline \multirow{5}{*}{$\begin{array}{l}\text { Healthcare } \\
\text { provider } \\
\text { communication }\end{array}$} & $\begin{array}{l}\text { Has your medical provider talked to you about whether you were interested in } \\
\text { becoming pregnant? }\end{array}$ & $20(36 \%)$ & $18(41 \%)$ & 0.68 \\
\hline & $\begin{array}{c}\text { Have you expressed an interest in becoming pregnant to your medical } \\
\text { provider? }\end{array}$ & $3(5 \%)$ & $19(43 \%)$ & $<0.0001$ \\
\hline & Has your medical provider talked to you about birth control or contraception? & $43(77 \%)$ & $28(64 \%)$ & 0.19 \\
\hline & $\begin{array}{l}\text { Has your medical provider talked to you about condoms to prevent STDs and } \\
\text { HIV transmission? }\end{array}$ & $52(93 \%)$ & $42(96 \%)$ & 0.69 \\
\hline & $\begin{array}{l}\text { Has your medical provider referred you to a reproductive specialist to discuss } \\
\text { methods to pursue pregnancy? }\end{array}$ & $4(7 \%)$ & $9(20 \%)$ & 0.07 \\
\hline
\end{tabular}




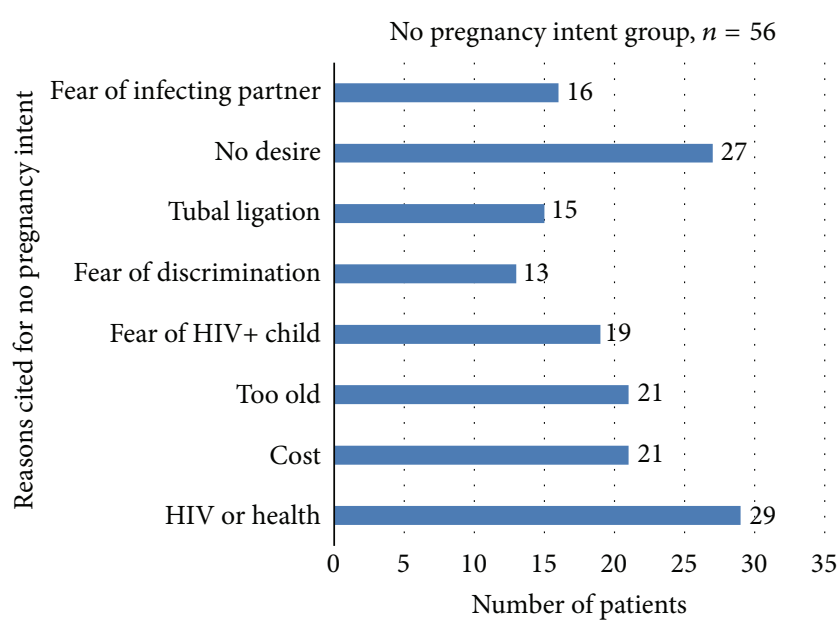

(a)

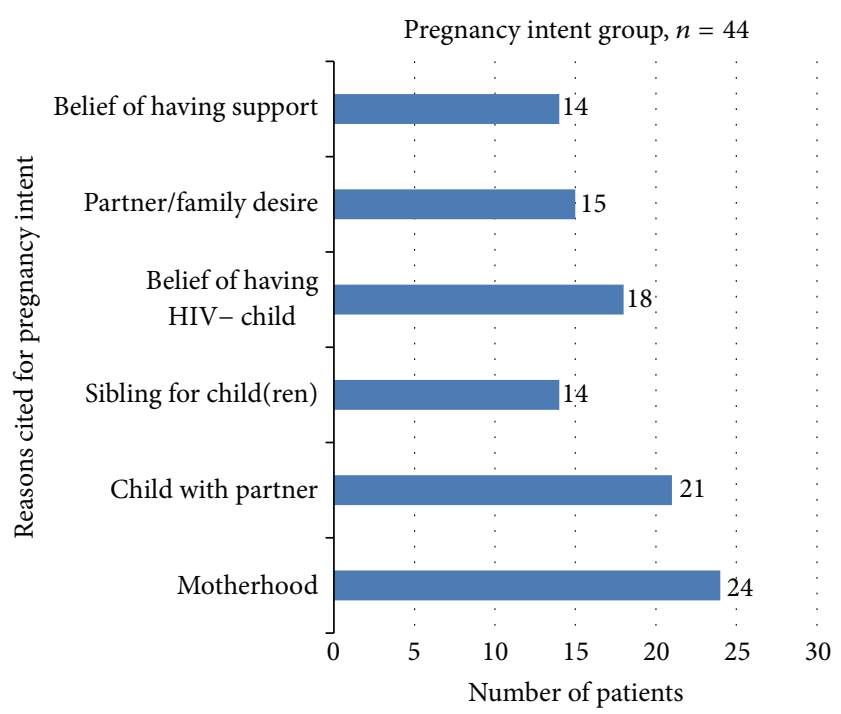

(b)

FIGURE 1: (a) Reasons for not desiring future pregnancy and (b) reasons for desiring future pregnancy.

referred to a reproductive specialist to discuss alternative methods of pregnancy.

Nondisclosure of HIV status to any partner in the prior year was relatively high (no desire $29 \%[n=14]$ versus desire $18 \%[n=7], p=0.53)$. Condom use rates and type of birth control in women with or without reproductive desire did not differ (Table 2).

Of 44 women with desire for future pregnancy, $84 \%$ thought they would have a future child and 10 women (23\%) currently reported condomless sex with the goal of pregnancy. Interestingly, two women (4\%) who did not report desire for a future pregnancy reported that they thought they would have a future child. Just under half of HIVinfected women reported that their HIV diagnosis impacted their pregnancy plans regardless of their desire for future pregnancy ( $45 \%$ versus $45 \%, p=1$ ). There was no difference $(p=0.71)$ in the knowledge-based MTCT question: "If you were to become pregnant, what is the probability that your child will contract HIV with proper medication and medical guidance?"

Provider rates of discussion were the highest for condom use (desire $96 \%$ versus no desire $93 \%, p=0.69$ ) followed by contraception use (desire 64\% and no desire 77\%, $p=$ 0.19 ) and the lowest in assessing desire for pregnancy (desire $41 \%$ versus no desire $35 \%, p=0.68$ ). Women desiring pregnancy were more likely to initiate a discussion with providers regarding pregnancy intent $(42 \%$ versus $5 \%, p \leq$ $0.0001)$.

Figure 1(a) highlights the reason(s) why women reported that they do not desire future children: HIV/health (52\%), no desire for children (48\%), cost (38\%), "too old" (38\%), and fear of an HIV positive child (34\%). Figure 1(b) reports the most common reason(s) to desire a future child: "experiencing motherhood" (55\%), wanting a child with the current partner (48\%), belief that the child will be HIV negative (41\%), and partner/family desire for child (34\%).
Logistic model results identified crude univariate predictors for desire of future pregnancy. Factors that reduced desire of future pregnancy include age (OR: $0.84,95 \%$ CI: $0.77-$ 0.90 ), number of prior pregnancies (OR: $0.82,95 \%$ CI: $0.68-$ 0.98 ), current children (OR: $0.26,95 \%$ CI: $0.08-0.81$ ), and time since HIV diagnosis (OR: $0.93,95 \%$ CI: $0.87-0.99$ ). Both current ART regimen (OR: 3.8, 95\% CI: 1.1-13.2) and partner desire for child (OR: 32.2, 95\% CI: 9.4-110.1) increased likelihood of desiring future pregnancy. Multivariate model significant predictors were age (AOR: 0.78 , 95\% CI: $0.68-$ 0.90 ) and partner's desire for child (AOR: $45.3,95 \%$ CI: 8.6238.0).

\section{Discussion}

Our study sample indicated that $44 \%$ of the reproductive aged HIV-infected women desire future pregnancy, $84 \%$ of whom think they will become pregnant. A majority of our sample reported serodiscordant relationships, and $21 \%$ reported not sharing HIV status with any sexual partner in the previous year. Women reported discussions with providers about condoms (94\%) and contraception (71\%) but discussed current or future specific reproductive desires less often (38\%). The reported lack of provider counseling for safe pregnancy planning could lead a patient to believe either that the provider has bias against pregnancy or that the patient should not become pregnant.

Our reported rate of reproductive desire is consistent with recent US studies $[4,6,7]$ but higher than earlier surveys [3]. This may be due to improvements in HIV treatment throughout the past decade. The rate of serodiscordance in the present study is higher than previously reported $[3,10]$ and notable as $38 \%$ of women reported that fear of infecting partner affected plans to pursue pregnancy. This highlights an opportunity for increased counseling on risk-reduction strategies among HIV-infected women. In our sample, $10 \%$ of 
all women reported current condomless sex with the goal of pregnancy and only $20 \%$ of women desiring future pregnancy reported referral to a reproductive specialist. This indicates the need for education about safer serodiscordant partner conception options. Recent surveys $[11,12]$ showed provider variability in awareness of and referrals for PrEP, timed unprotected intercourse, intrauterine insemination (IUI), and in vitro fertilization (IVF) highlighting the need for provider training on these issues.

Our final multivariate model included age and partner desire for children as significant predictors of desire for future pregnancies. There are a variety of significant predictors in previous models, with age being the most consistent $[3,4,13$, 14]. Number of children, length of time since diagnosis, and ART use were significant in a few models, but we presume these terms are collinear with age in our model. Partner's desire for children is a strong influence on reproductive desires of women in the US $[4,14]$ and significant in our model. This multivariate analysis is significant as it suggests that HIV-infected women are making decisions regarding future pregnancies based on similar factors to HIV-negative women.

Limitations of this study include a small sample size and cross-sectional study design which is not able to capture evolving trends in pregnancy desire among HIV-infected women. However, we were able to include a diverse representation of race and age in this cohort and did attempt to capture both prior and future intent of pregnancy among HIVinfected women. Recruitment of women in their forties could be viewed as a limitation when generalizing to a younger cohort; however, $18 \%$ of women above forty reported desire for pregnancy and contributed to descriptive endpoints.

\section{Conclusions}

It is important that each HIV-infected woman receive comprehensive reproductive health counseling, including assessment of pregnancy desire with defined discussions on reproductive intent. These conversations can be an opportunity to identify serodiscordant couples, assess disclosure of HIV status to sexual partners, and initiate education regarding safer options to avoid or reduce partner and vertical transmission such as PrEP, timed unprotected intercourse, IUI, and IVF. Incorporating training modules for providers managing reproductive aged HIV-infected women's HIV and overall primary care could improve counseling rates and reduce HIV transmission risk.

\section{Competing Interests}

The authors declare that there are no competing interests regarding the publication of this paper.

\section{Acknowledgments}

The authors acknowledge Varina Clark, Brittany Katz, Eliza DeCroce-Movson, Ihiechi Ohia-Enyia, and Patricia Delgado for recruiting assistance. This research was supported by the Lifespan/Tufts/Brown Center for AIDS Research (P30AI042853) and supporting grants from NIH (Aadia I. Rana, 1K23MH100955), HRSA (Corinne M. Rhodes, T32HP1025), and the Tyoichi Sasakawa Fellowship Fund (Corinne M. Rhodes).

\section{References}

[1] Centers for Disease Control and Prevention, "Monitoring selected national HIV prevention and care objectives by using HIV surveillance data-United States and 6 dependent areas2012," HIV Surveillance Supplemental Report, vol. 19, no. 3, 2014, http://www.cdc.gov/hiv/library/reports/surveillance/.

[2] J. M. McMahon, J. E. Myers, A. E. Kurth et al., "Oral preexposure prophylaxis (PrEP) for prevention of HIV in serodiscordant heterosexual couples in the United States: opportunities and challenges," AIDS Patient Care and STDs, vol. 28, no. 9, pp. 462-474, 2014.

[3] J. L. Chen, K. A. Phillips, D. E. Kanouse, R. L. Collins, and A. Miu, "Fertility desires and intentions of HIV-positive men and women," Family Planning Perspectives, vol. 33, no. 4, pp. 144-165, 2001.

[4] S. Finocchario-Kessler, J. K. Dariotis, M. D. Sweat et al., "Do HIV-infected women want to discuss reproductive plans with providers, and are those conversations occurring?" AIDS Patient Care and STDs, vol. 24, no. 5, pp. 317-323, 2010.

[5] M. Y. Sutton, E. Frazier, W. Short, and J. Skarbinski, "Pregnancies among reproductive-aged human immunodeficiency virus-infected women in care, United States," Obstetrics \& Gynecology, vol. 123, supplement 1, pp. 33S-34S, 2014.

[6] S. Finocchario-Kessler, M. D. Sweat, J. K. Dariotis et al., "Understanding high fertility desires and intentions among a sample of urban women living with HIV in the united states," AIDS and Behavior, vol. 14, no. 5, pp. 1106-1114, 2010.

[7] S. Finocchario-Kessler, N. Mabachi, J. K. Dariotis, J. Anderson, K. Goggin, and M. Sweat, "We weren't using condoms because we were trying to conceive': the need for reproductive counseling for HIV-positive women in clinical care," AIDS Patient Care and STDs, vol. 26, no. 11, pp. 700-707, 2012.

[8] Z. Bursac, C. H. Gauss, D. K. Williams, and D. W. Hosmer, "Purposeful selection of variables in logistic regression," Source Code for Biology and Medicine, vol. 3, article 17, 2008.

[9] Panel on Antiretroviral Guidelines for Adults and Adolescents, Guidelines for the Use of Antiretroviral Agents in HIV-1-Infected Adults and Adolescents, Department of Health and Human Services, 2016, http://www. aidsinfo.nih.gov/ContentFiles/AdultandAdolescentGL.pdf.

[10] L. Rahangdale, A. Stewart, R. D. Stewart et al., "Pregnancy intentions among women living with HIV in the United States," Journal of Acquired Immune Deficiency Syndromes, vol. 65, no. 3, pp. 306-311, 2014.

[11] M. L. Scherer, N. C. Douglas, B. H. Churnet et al., "Survey of HIV care providers on management of HIV serodiscordant couples-assessment of attitudes, knowledge, and practices," AIDS Care: Psychological and Socio-Medical Aspects of AIDS/ HIV, vol. 26, no. 11, pp. 1435-1439, 2014.

[12] M. A. Lampe, D. K. Smith, G. J. E. Anderson, A. E. Edwards, and S. R. Nesheim, "Achieving safe conception in HIV-discordant couples: the potential role of oral preexposure prophylaxis (PrEP) in the United States," American Journal of Obstetrics and Gynecology, vol. 204, no. 6, pp. 488.el-488.e8, 2011. 
[13] N. L. Stanwood, S. E. Cohn, J. R. Heiser, and M. Pugliese, "Contraception and fertility plans in a cohort of HIV-positive women in care," Contraception, vol. 75, no. 4, pp. 294-298, 2007.

[14] R. L. Sowell, C. L. Murdaugh, C. L. Addy, L. Moneyham, and A. Tavokoli, "Factors influencing intent to get pregnant in HIVinfected women living in the southern USA," AIDS Care, vol. 14, no. 2, pp. 181-191, 2002. 


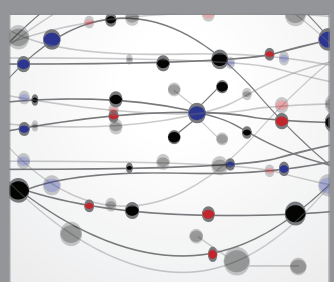

The Scientific World Journal
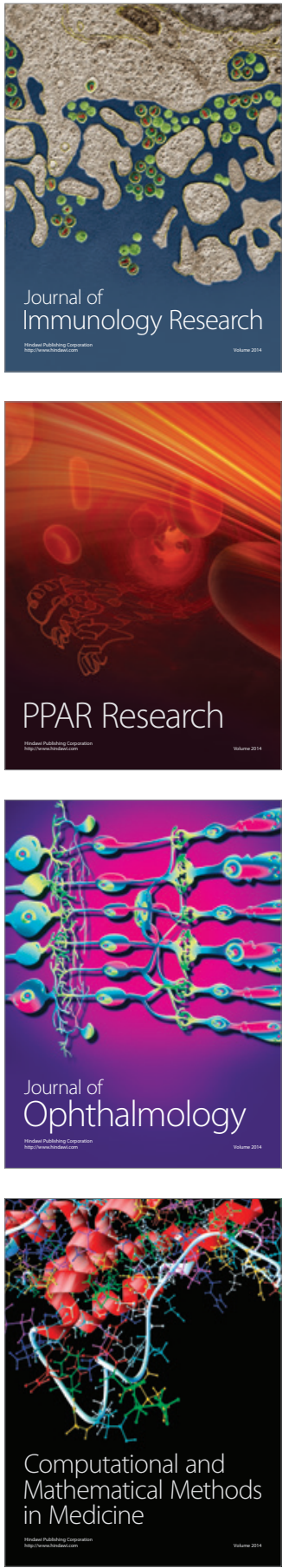

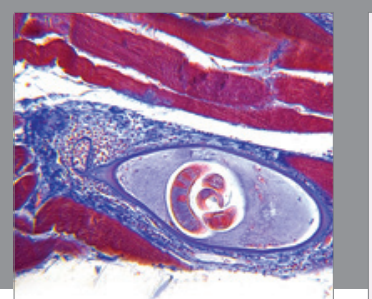

Gastroenterology Research and Practice

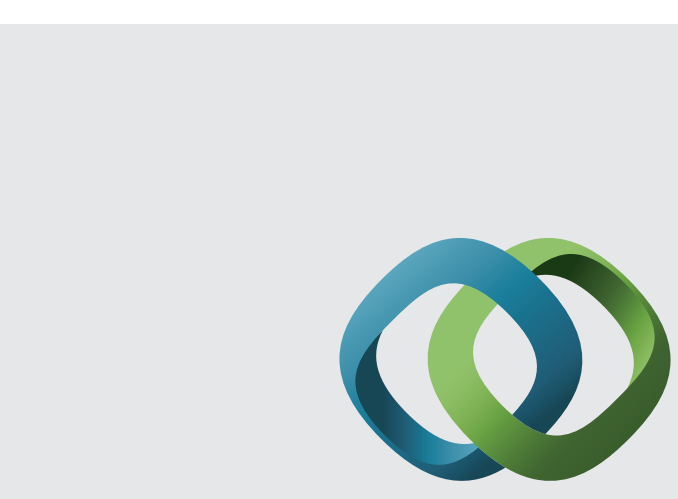

\section{Hindawi}

Submit your manuscripts at

http://www.hindawi.com
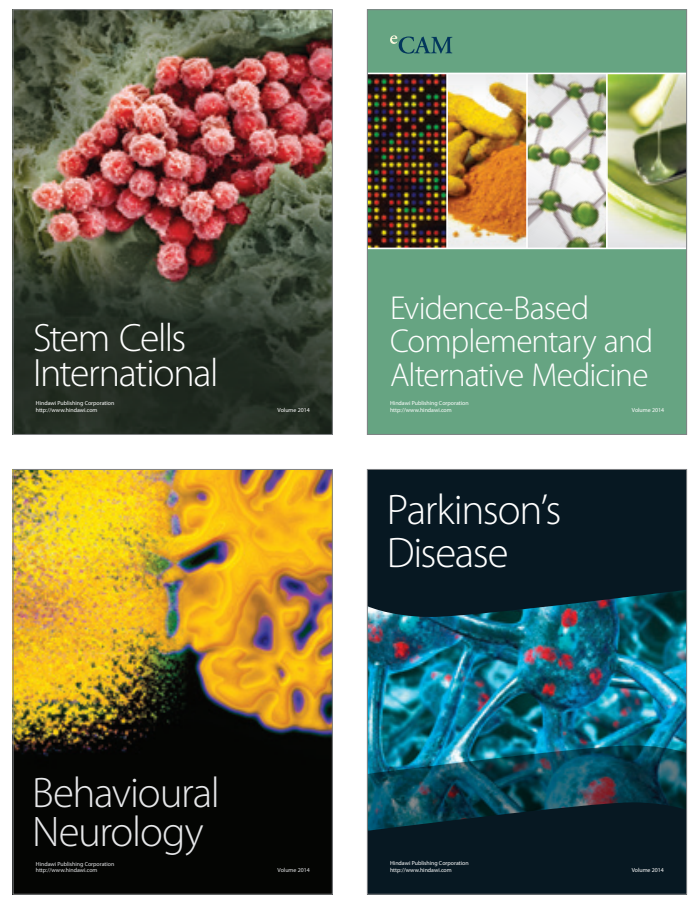
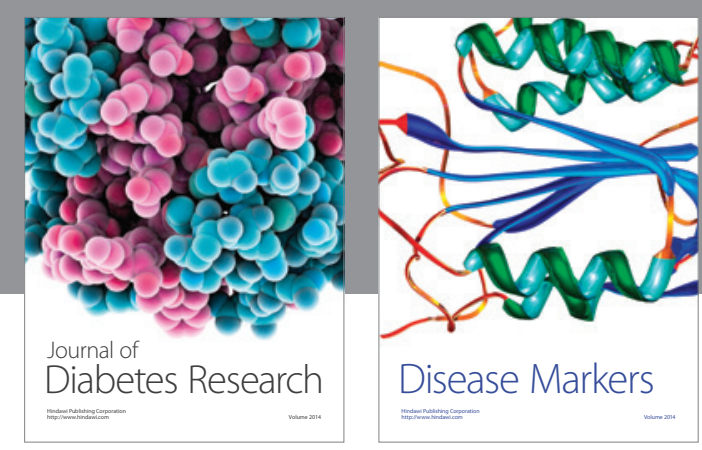

Disease Markers
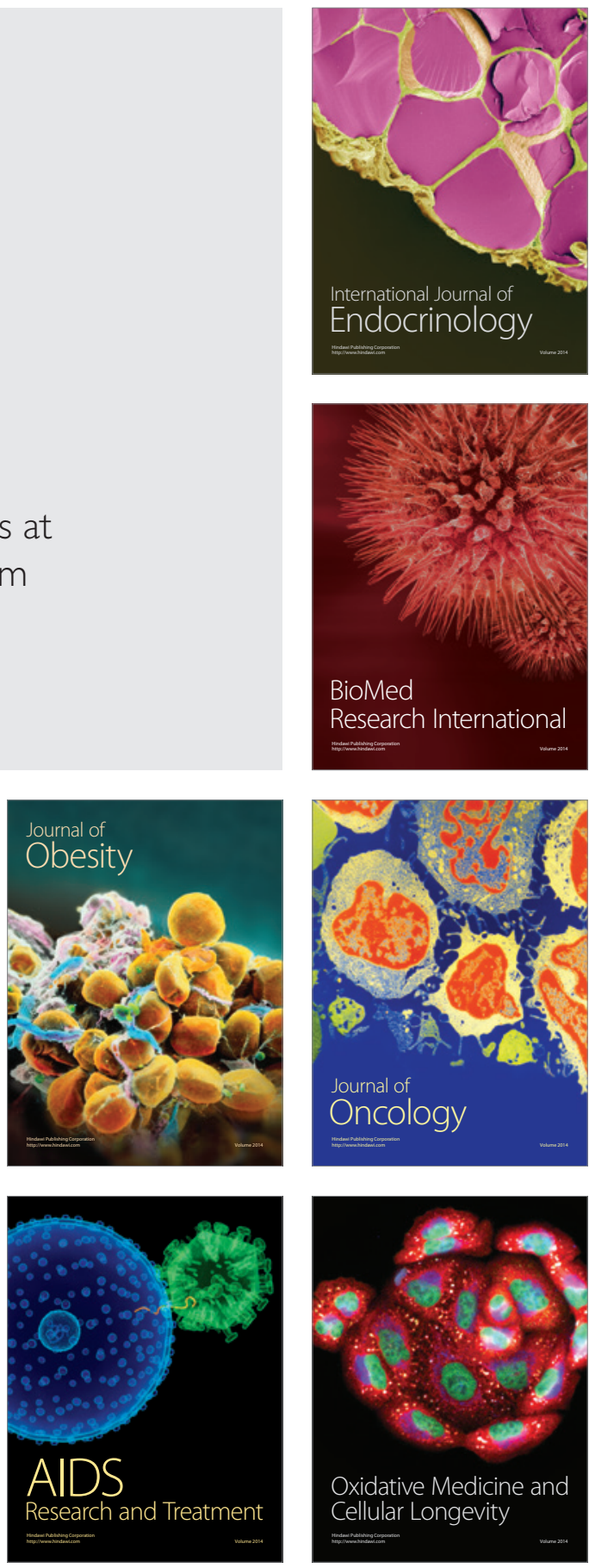